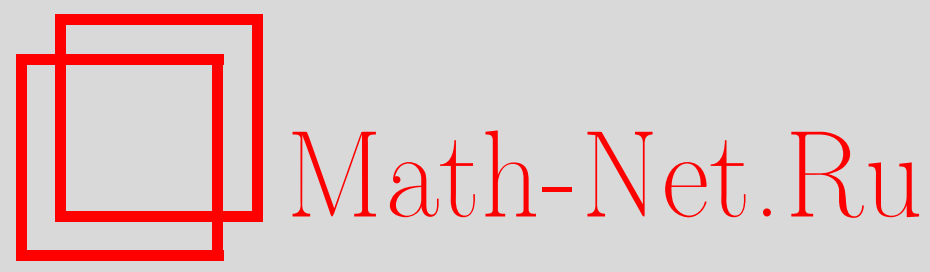

В. Н. Суриков, Время жизни случайной двоичной последовательности (параллельной системы), Дискрет. матем., 2010, том 22, выпуск 2, 51-59

DOI: https://doi.org/10.4213/dm1094

Использование Общероссийского математического портала Math-Net.Ru подразумевает, что вы прочитали и согласны с пользовательским соглашением http://www.mathnet.ru/rus/agreement

Параметры загрузки:

IP : 3.95 .254 .165

26 апреля 2023 г., 03:12:59 
УДК 519. 2

\title{
Время жизни случайной двоичной последовательности (параллельной системы)
}

\author{
() 2010 г. В. Н. Суриков
}

\begin{abstract}
В статье вводится и изучается вероятностная модель параллельной системы, состоящей из $m$ элементов и предназначенная для выполнения однородных заданий. На первом этапе на каждый элемент системы поступает одно задание из общего числа $n$ заданий. Результатом работы элемента является либо исполнение задания, либо отказ элемента. В случае отказа элемент исключается из системы, а невыполненное задание возвращается в очередь заданий, ожидающих исполнения. На втором этапе в систему направляется $m_{1}$ заданий, где $m_{1}-$ число элементов системы, оставшихся исправными после первого этапа, и так далее. В статье проведен подробный анализ предложенной модели в случае, когда каждый элемент системы выполняет задание независимо от остальных элементов с одинаковой вероятностью.
\end{abstract}

\section{1. Определения}

Рассмотрим двоичную (бинарную) последовательность

$$
x_{1}, x_{2}, \ldots,
$$

в которой $x_{i} \in\{0,1\}, i=1,2, \ldots$ Можно установить взаимно однозначное соответствие между последовательностями (1) и последовательностями из множества целых неотрицательных чисел, расширенного элементом $+\infty$,

$$
X_{1}, X_{2}, \ldots,
$$

полагая $X_{1}$ равным числу знаков в последовательности (1) до первого нуля, $X_{t}, t \geqslant 2$, равным числу единиц между $(t-1)$-м и $t$-м вхождениями нуля в последовательности (1).

Зафиксируем натуральное число $m \geqslant 2$ и образуем из (1) последовательности двоичных векторов

$$
\xi_{1}, \xi_{2}, \ldots
$$

и целых неотрицательных чисел

$$
m_{1}, m_{2}, \ldots,
$$

следующим рекуррентным образом. Первые члены последовательностей (3) и (4) равны $\xi_{1}=\left(x_{1}, \ldots, x_{m}\right), m_{1}=\left\|\xi_{1}\right\|$ соответственно. Символом $\|v\|$ будем обозначать число единиц (вес) двоичного вектора $v$. 
При $m_{1}=0$ процесс построения последовательности (3) обрывается и $m_{t}=0$, $t=1,2, \ldots$ При $m_{1}>0, \xi_{2}=\left(x_{m+1}, \ldots, x_{m+m_{1}}\right), m_{2}=\left\|\xi_{2}\right\|$, и т. д.

При $m_{t}=0$ процесс построения последовательности (3) обрывается и $m_{t}=m_{t+1}=$

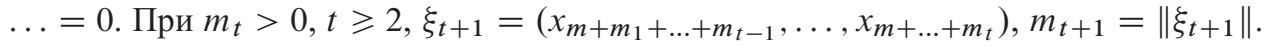

Другими словами, мы разбиваем последовательность (1) на участки следующим образом. Первый участок имеет размер $m$. Размер следующего вектора равен числу единиц в предыдущем участке последовательности (1) и так далее, до тех пор, пока не появится участок последовательности (1), состоящий только из нулей, или в (1) менее $m$ нулей и последовательности (3) и (4) будут иметь неограниченную длину.

Вектор $\xi_{t}, t=1,2, \ldots$, будем называть $t$-м циклом жизни последовательности (1). Обобщенную функцию (допускающую значение $+\infty$ )

$$
R^{(m)}=m_{1}+m_{2}+\ldots
$$

будем называть ресурсом последовательности (1).

Нетрудно видеть, что

$$
R^{(m)}=X_{1}+\ldots+X_{m}
$$

Обозначим через $S_{k}, k=1,2, \ldots$, и $R_{t}, t=1,2, \ldots$, суммы первых $k$ и $t$ членов последовательностей (2) и (4), соответственно.

Для целого $n, 1 \leqslant n \leqslant S_{m}$, определим траектории процессов восстановления

$$
\begin{aligned}
& v_{n}\left(X_{1}, \ldots, X_{m}\right)=v_{n}=\min \left\{k: S_{k} \geqslant n\right\}, \\
& \tau_{n}\left(X_{1}, \ldots, X_{m}\right)=\tau_{n}=\min \left\{t: R_{t} \geqslant n\right\} .
\end{aligned}
$$

Из определений следует, что $\{n / m\} \leqslant \tau_{n} \leqslant n$, где $\{x\}$ здесь и всюду далее обозначает наименьшее целое число, не меньшее $x$ (округление до целого числа сверху), $\{x\} \geqslant[x]$, где $[x]$ - целая часть числа $x$.

Функцию $\tau=\tau_{S_{m}}$ будем называть временем жизни последовательности (1). Поведение функции $\tau_{n}$ описывается в следующем утверждении.

Лемма 1. При $v_{n}=1$

$$
\tau_{n}=\{n / m\} .
$$

При $v_{n}=k, 2 \leqslant k \leqslant m$,

$$
0 \leqslant \tau_{n}^{(m)}-\frac{X_{1}}{m}-\ldots-\frac{X_{k-1}}{m-k+2}-\frac{n-S_{k-1}}{m-k+1} \leqslant\left\{\frac{1}{m}+\ldots+\frac{1}{m-k+2}\right\}
$$

Доказательство. При $v_{n}=1$ соотношение (7) следует из неравенства (5) $S_{1} \geqslant n$.

Обозначим через $r_{i}$ остатки от деления $X_{i}$ на $m-i+1$.

Тогда $X_{1}-r_{1}$ кратно $m$, в $\left(\left(X_{1}-r_{1}\right) / m+1\right)$-ом цикле есть нуль. Это означает, что последовательность (1), начиная с $\left(X_{1}-r_{1}+1\right)$-го члена, разбивается на циклы с весом, не большим $m-1$. Это влечет равенство

$$
\tau_{n}^{(m)}\left(X_{1}, \ldots, X_{m}\right)=\frac{X_{1}-r_{1}}{m}+\tau_{n-X_{1}+r_{1}}^{(m-1)}\left(X_{2}+r_{1}, \ldots, X_{m}\right),
$$

так как $n>X_{1}$. 
Пусть $k=2$. В этом случае

$$
\tau_{n-X_{1}+r_{1}}^{(m-1)}\left(X_{2}+r_{1}, \ldots, X_{m}\right)=\left\{\frac{n-X_{1}+r_{1}}{m-1}\right\}
$$

и

$$
\tau_{n}^{(m)}\left(X_{1}, \ldots, X_{m}\right)=\left\{\frac{X_{1}-r_{1}}{m}+\frac{n-X_{1}+r_{1}}{m-1}\right\}=\left\{\frac{X_{1}}{m}+\frac{n-X_{1}}{m-1}+\frac{r_{1}}{m(m-1)}\right\} .
$$

Следуя принципу математической индукции, предположим, что для любого $k>2$ верно соотношение

$$
\begin{aligned}
\tau_{n}^{(m)}\left(X_{1}, \ldots, X_{m}\right)=\left\{\frac{X_{1}}{m}+\ldots+\frac{X_{k-1}}{m-k+2}+\frac{n-S_{k-1}}{m-k+1}\right. & \\
& \left.\quad+\frac{r_{1}}{m(m-1)}+\ldots+\frac{r_{k-1}}{(m-k+1)(m-k)}\right\} .
\end{aligned}
$$

Тогда, продолжая равенство (9), получаем, что

$$
\begin{aligned}
\tau_{n}^{(m)}\left(X_{1}, \ldots, X_{m}\right) & =\frac{X_{1}-r_{1}}{m}+\left\{\frac{X_{2}+r_{1}}{m-1}+\frac{r_{2}}{(m-1)(m-2)}+\ldots\right\} \\
& =\left\{\frac{X_{1}}{m}+\frac{r_{1}}{m(m-1)}+\frac{X_{2}}{m-1}+\frac{r_{2}}{(m-1)(m-2)}+\ldots\right\} .
\end{aligned}
$$

Эта цепочка равенств доказывает справедливость (10). Учитывая, что $0 \leqslant r_{i} \leqslant m-i$, из (10) получим неравенства

$$
\begin{aligned}
\frac{X_{1}}{m}+\ldots+\frac{n-S_{k-1}}{m-k+1} & \leqslant\left\{\frac{X_{1}}{m}+\ldots+\frac{X_{k-1}}{m-k+2}+\frac{n-S_{k-1}}{m-k+1}\right\} \leqslant \tau_{n}, \\
\tau_{n} & \leqslant\left\{\frac{X_{1}+1}{m}+\ldots+\frac{X_{k-1}+1}{m-k+2}+\frac{n-S_{k-1}}{m-k+1}\right\} \\
& \leqslant\left\{\frac{X_{1}}{m}+\ldots+\frac{X_{k-1}}{m-k+2}+\frac{n-S_{k-1}}{m-k+1}\right\}+\left\{\frac{1}{m}+\ldots\right\} .
\end{aligned}
$$

Из второй цепочки неравенств (11) получаем, что

$$
0 \leqslant\left\{\tau_{n}-\frac{X_{1}}{m}-\ldots\right\} \leqslant\left\{\frac{1}{m}+\ldots\right\} .
$$

Отсюда, с учетом неравенств из первой цепочки (11), получаем утверждение леммы.

Обратим внимание на то, что нижняя и верхняя границы для $\tau_{n}$ в $(11)$ достижимы при соответствующем выборе $X_{1}, \ldots, X_{k-1}$ и $n$.

\section{2. Иллюстрации}

Для иллюстрации введенных определений рассмотрим пример, который ранее обсуждался в [1].

Параллельная система из $m$ элементов применяется для выполнения заданий. Каждое задание направляется на выполнение одному элементу. Результатом выполнения является 
либо исполненное задание, либо отказ элемента. Это отражается символом 1 или 0 в последовательности результатов выполнения заданий (1). В случае отказа элемента задание направляется в последовательность невыполненных заданий на первое место. Элемент, отказавший в исполнении задания, исключается из системы.

На первом этапе в систему направляется $m$ заданий. Результатом их исполнения является вектор $\xi_{1}$. На втором этапе формируется комплект размером $m_{1}$, равный числу выполненных заданий на первом этапе, и т. д.

Участок последовательности (1), который был назван $t$-м циклом, отражает результат выполнения заданий, направленных в систему на $t$-м этапе.

Особый интерес представляет круг вопросов, связанный с применением параллельных систем для решения задачи, допускающей разбиение на $n$ независимых заданий. Исследователей интересует положительный исход, то есть решение задачи до отказа всей системы.

В связи с этим наибольшую значимость приобретают такие характеристики, как среднее время решения задачи, которое может измеряться числом этапов до достижения результата, а также число отказавших в процессе решения задачи элементов системы.

Для их количественных оценок можно использовать теоретико-вероятностные модели, задавая различные вероятностные пространства $(\Omega, \sigma, \mathbf{P})$ для значений вектора $\left(X_{1}, \ldots, X_{m}\right)$.

Рассматриваемые характеристики широко обсуждаются специалистами по математическим методам в теории надежности [2]. Основная часть исследований посвящена изучению моделей, в которых надежность и время жизни системы являются функциями от вектора времен ожидания отказов элементов системы $\left(Y_{1}, \ldots, Y_{m}\right)$ [3-6], для которого также задается соответствующее вероятностное пространство. Содержательные результаты, представленные в этих работах, относятся к случаю, когда компоненты вектора отказов элементов являются непрерывными и перестановочными случайными величинами.

Изучение поведения системы при дискретном распределении этого вектора даже в случае, когда компоненты являются независимыми одинаково распределенными случайными величинами, приводит к сложностям, которые можно преодолеть переходом к вектору $\left(X_{1}, \ldots, X_{m}\right)$. Существует взаимно-однозначная связь между векторами $\left(Y_{1}, \ldots, Y_{m}\right)$ и $\left(X_{1}, \ldots, X_{m}\right)$, которую легко проследить при помощи следующей конструкции.

Рассмотрим случайную матрицу, состоящую из $m$ строк. Каждая строка состоит из нулей и единиц и имеет, по крайней мере, один нуль. Заменим в каждой строке все элементы после первого появления нуля на символ $f$ (отказ). Можно трактовать число единиц в $i$-й строке до символа $f$ как время ожидания отказа $i$-го элемента, а число единиц в $t$-м столбце матрицы как число выполненных заданий $m_{t}$ в $t$-м цикле.

Тогда время жизни системы, ее ресурс и темпы его реализации можно изучать либо как функцию от вектора $\left(Y_{1}, \ldots, Y_{m}\right)$, что сделано в [3-6], либо как процесс восстановления для марковских последовательностей $m_{t}, t=1,2, \ldots$

Для осуществления перехода от пространства состояний элементов системы к пространству результатов ее функционирования выпишем элементы матрицы в одну строку последовательно по столбцам до $m$-го нуля и удалим из этой строки все символы $f$. В полученной таким образом двоичной последовательности место первого появления 0 можно трактовать как первый отказ в исполнении заданий (а не отказ элемента системы) и т. д.

Обсуждение методологических аспектов соответствия математических моделей приложениям в теории надежности выходит за рамки этой работы. Вопросы выбора моделей для реальных систем освещались в [7] и [8].

Работа посвящена изучению функции восстановления процесса $\tau_{n}, n=1,2, \ldots$, при некоторых предположениях о распределении случайных величин $\left(X_{1}, \ldots, X_{m}\right)$. 
В том случае, когда элементы рассматриваемой матрицы независимы и одинаково распределены, все три подхода равноправны и приводят к одинаковым результатам для времени ожидания выполнения задачи с $n$ заданиями.

\section{3. Произвольное распределение мест сбоев}

Случайные места сбоев $x_{i}, i=1,2, \ldots$, принимают лишь положительные значения. Поэтому их математические ожидания, возможно бесконечные, всегда существуют. В тех случаях, когда средний ресурс системы

$$
\mathbf{M} R^{(m)}=\mathbf{M} X_{1}+\ldots+\mathbf{M} X_{m}
$$

значительно превосходит единицу, хорошим приближением для времени жизни системы является случайная величина

$$
L=L\left(X_{1}, \ldots, X_{m}\right)=\sum_{k=1}^{m} \frac{X_{k}}{m-k+1}
$$

Это следует из теоремы 1 , которая является непосредственным следствием доказанной выше леммы.

Теорема 1. Для математического ожидания времени жизни двоичной последовательности (1) справедливы неравенства

$$
0 \leqslant \mathbf{M} \tau-\mathbf{M} L \leqslant\left\{\frac{1}{2}+\ldots+\frac{1}{m}\right\} .
$$

Для среднего времени выполнения задачи с $n$ заданиями при условии, что ресурс системы $R^{(m)} \geqslant n$, справедливы неравенства, представленные в следующем утверждении.

Теорема 2. Для произвольного распределения величин $\left(X_{1}, X_{2}, \ldots, X_{m}\right)$ и положительного числа п справедливы неравенства

$$
\begin{aligned}
0 \leqslant & \sum_{t \geqslant n / m} t \mathbf{P}\left\{\tau_{n}=t, R^{(m)} \geqslant n\right\} \\
& \quad-\sum_{k=1}^{m} \frac{1}{m-k+1}\left(n+\sum_{\nu=0}^{k-1} \frac{k-v}{m-v+1} \mathbf{M}\left(X_{\nu} \mid v_{n}=k\right)\right) \mathbf{P}\left\{v_{n}=k\right\} \\
\leqslant & \sum_{k=1}^{m-1}\left\{\delta_{m}(k)\right\} \mathbf{P}\left\{v_{n}=k\right\},
\end{aligned}
$$

где $X_{0}=0, \delta_{m}=\delta_{m}=1 / m u$

$$
\delta_{m}(k)=\sum_{\nu=1}^{k-1} \frac{1}{m-v+1}, \quad k>2 .
$$




\section{4. Последовательность испытаний Бернулли}

Пусть (1) является последовательностью независимых случайных величин с вероятностями

$$
\mathbf{P}\left\{x_{1}=0\right\}=p, \quad \mathbf{P}\left\{x_{1}=1\right\}=1-p, \quad 0<p<1 .
$$

В этом случае $X_{i}$ равна времени ожидания появления нуля в последовательности испытаний Бернулли и имеет геометрическое распределение с параметром $p$ (см. [9])

$$
\mathbf{P}\left\{X_{i}=k\right\}=p(1-p)^{k}, \quad k=0,1, \ldots
$$

Последовательность целочисленных случайных величин (4) образует однородную цепь Маркова с вектором начальных распределений

$$
\mathbf{P}\left\{m_{1}=k\right\}=\left(\begin{array}{l}
m \\
k
\end{array}\right)(1-p)^{k} p^{m-k}, \quad k=0,1, \ldots, m,
$$

и вероятностями переходов

$$
\mathbf{P}\left\{m_{i+1}=k \mid m_{i}=l\right\}=\left(\begin{array}{l}
l \\
k
\end{array}\right)(1-p)^{k} p^{l-k}, \quad k=0,1, \ldots, m .
$$

Здесь полагаем $\left(\begin{array}{l}l \\ k\end{array}\right)=0$ при $l<k$. Распределение (4) совпадает в данном случае с распределением последовательности случайных величин

$$
m_{1}^{\prime}, m_{2}^{\prime}, \ldots,
$$

в которой

$$
m_{i}^{\prime}=\sum_{\nu=1}^{m} \mathbf{I}\left(Y_{v}<i\right)
$$

$\mathbf{I}(A)$ обозначает индикатор события $A, Y_{1}, Y_{2}, \ldots$ - последовательность независимых одинаково распределенных случайных величин, каждая из которых имеет геометрическое распределение с параметром $p$.

Такая вероятностная схема рассматривалась в [1], где было анонсировано утверждение, частично аналогичное утверждению следующей теоремы.

Теорема 3. Для последовательности испытаний Бернулли (1) с вероятностями

$$
\mathbf{P}\left\{x_{i}=0\right\}=p, \quad \mathbf{P}\left\{x_{i}=1\right\}=1-p, \quad 0<p<1,
$$

и цельх чисел $n \geqslant m \geqslant 2$ имеют место следующие утверждения.

(1) Математическое ожидание ресурса последовательности (1) равно

$$
\mathbf{M} R^{(m)}=m(1-p) p .
$$

(2) Математическое ожидание $\mathbf{M} \tau$ времени жизни последовательности (1) не меньше, чем

$$
\frac{1-p}{p}\left(\frac{1}{m}+\frac{1}{m-1}+\ldots+1\right)
$$

и не больше, чем

$$
\left(\frac{1-p}{p}+1\right)\left(\frac{1}{m}+\frac{1}{m-1}+\ldots+1\right) .
$$


(3) Вероятность решения задачи с $n$ заданиями до сбоя системы равна

$$
\begin{aligned}
\mathbf{P}(n, m, p) & =\mathbf{P}\left\{R^{(m)} \geqslant n\right\}=(1-p)^{n} \sum_{k=0}^{m-1}\left(\begin{array}{c}
n+k-1 \\
k
\end{array}\right) p^{k} \\
& =(1-p)^{n} \sum_{\nu=0}^{m-1}\left(\begin{array}{c}
n+m-1 \\
v
\end{array}\right) p^{v}(1-p)^{m-1+v} .
\end{aligned}
$$

(4) Для математического ожидания времени решения задачи с $n$ заданиями справедливы неравенства

$$
\begin{aligned}
0 \leqslant & \mathbf{P}\left\{R^{(m)} \geqslant n\right\} \mathbf{M}\left(\tau_{n} \mid R^{(m)} \geqslant n\right) \\
& \quad-\sum_{k=1}^{m}\left(\frac{1}{k(m-k+1)}+\frac{(n-1)}{m} \varphi_{m}(k)\right)\left(\begin{array}{c}
n+k-2 \\
k-1
\end{array}\right) p^{k-1}(1-p)^{n} \\
\leqslant & \sum_{k=1}^{m-1} \delta_{m}(k)\left(\begin{array}{c}
n+k-2 \\
k-1
\end{array}\right) p^{k-1}(1-p)^{n},
\end{aligned}
$$

где

$$
\varphi_{m}(k)=\frac{m}{k} \sum_{\nu=1}^{k} \frac{1}{m-v+1}, \quad k=1, \ldots, m,
$$

a $\delta_{m}(k)$ определено в теореме 2.

Доказательство. Соотношения (18), (19) и (20) следуют из формул (12), (13) и свойств геометрического распределения. В условиях теоремы случайная величина $R^{(m)}$ имеет отрицательное биномиальное распределение с параметрами ( $p, m)$ (см. [9], с. 436)

$$
\begin{aligned}
\mathbf{P}\left\{R^{(m)}=k\right\} & =\mathbf{P}\left\{X_{1}+\ldots+X_{m}=k\right\} \\
& =\left(\begin{array}{c}
m+k-1 \\
k
\end{array}\right) p^{m}(1-p)^{k}, \quad k=0,1,2, \ldots
\end{aligned}
$$

Событие $\left\{v_{n}=k\right\}$ означает, что в $(n+k-1)$-м испытании последовательности (1) появилась единица, а в первых $n+k-2$ появились $n-1$ единица и $k-1$ нуль. Это, в свою очередь, означает, что

$$
\mathbf{P}\left\{v_{n}=k\right\}=\left(\begin{array}{c}
n+k-2 \\
k-1
\end{array}\right) p^{k-1}(1-p)^{n}, \quad k=1,2, \ldots
$$

Поскольку

$$
\left\{R^{(m)} \geqslant n\right\}=\bigcup_{k=1}^{m-1}\left\{v_{n}=k\right\},
$$

из этого соотношения вытекает справедливость первого равенства (21).

Аналитическое доказательство правого равенства в (21) можно провести при помощи соотношения

$$
x \sum_{\nu \geqslant N}\left(\begin{array}{l}
v \\
n
\end{array}\right) x^{M}(1-x)^{v}=\sum_{\nu=0}^{M}\left(\begin{array}{c}
N \\
v
\end{array}\right) x^{v}(1-x)^{N-v}, \quad 1 \leqslant m \leqslant N, \quad 0 \leqslant x \leqslant 1,
$$


которое доказано в [10]. Существует целый ряд классических вероятностных доказательств правой части (21), ссылки на которые можно найти в [11]. Они основаны на следующем факте, наиболее подробно описанном в [12].

Пусть $B_{N}$ - число успехов в первых $N$ испытаниях (1), $T_{k}-$ минимальное число испытаний, необходимых для получения ровно $k$ неудач, $U_{n}-$ минимальное число испытаний, необходимых для получения $n$ успехов. Тогда

$$
\mathbf{P}\left\{B_{N} \geqslant N-k\right\}=\mathbf{P}\left\{T_{k}>N\right\}=\mathbf{P}\left\{U_{N-k} \leqslant N\right\} .
$$

Хорошо известно (см., например, [13], глава 6, раздел 8), что $T_{k}$ имеет отрицательное биномиальное распределение.

В условиях теоремы распределение вектора $\left(X_{1}, X_{2}, \ldots, X_{k-1}, n-1-S_{k-1}\right)$ при условии $v_{n}=k$ совпадает с распределением координат вектора решений уравнения $y_{1}+\ldots+y_{k}=n-1$ в неотрицательных целых числах при равномерном распределении на множестве всех решений, которое является частным случаем обобщенной схемы размещения частиц по ячейкам [14]. Следовательно,

$$
\mathbf{M}\left(X_{v}, v_{n}=k\right)=\mathbf{M} y_{v}=\frac{n-1}{k}, \quad v=1, \ldots, k-1 .
$$

Справедлива цепочка равенств

$$
\begin{aligned}
& \frac{1}{n-k+1}\left(n+\sum_{\nu=0}^{k-1} \frac{k-v}{(m-v+1)} \mathbf{M}\left(X_{v} \mid v_{n}=k\right)\right) \\
& \quad=\frac{1}{m-k+1}\left(n+\frac{1}{k} \sum_{\nu=0}^{k-1} \frac{(k-v)(n-1)}{(m-v+1)}\right)=\frac{1}{k(m-k+1)}+\frac{(n-1)}{m} \varphi_{m}(k) .
\end{aligned}
$$

Подставляя полученные значения в (14), завершим доказательство теоремы.

Автор выражает признательность А. Ф. Ронжину за внимание к работе и ряд конструктивных замечаний. В частности, им предложена формулировка леммы 1 , которая существенно расширяет границы применимости основных результатов работы.

\section{Список литературы}

1. Ронжин А. Ф., Суриков В. Н., О времени полного перебора. Обозрение прикладной и промышленной математики (2007) 14, №4, 506-508.

2. Samaniego F. J., On closure of the IFR class under formation of coherent systems. IEEE Trans. Reliab. (1985) 34, 69-72.

3. Navarro J., Balakrishnan N., Samaniego F. J., Mixture representations of residual lifetimes of used systems. J. Appl. Probab. (2008) 45, 1097-1112.

4. Navarro J., Rychlik T., Reliability and expectation bounds for coherent systems with exchangeable components. J. Multivariate Anal. (2007) 98, 102-113.

5. Spizzichino F., Ageing and positive dependence. In: Encyclopaedia of Statistics for Quality and Reliability. Wiley, Chichester, 2007, pp. 82-95.

6. Седякин Н. М., Об одном физическом принципе теории надежности. Известия АН СССР, Техническая кибернетика (1966), № 3, 80-87. 
7. Багдонавичюс В. Б., Статистическая проверка модели аддитивного накопления повреждений. Теория вероятностей и ее применения (1978) 23, №2, 403-408.

8. Вероятность и математическая статистика. В сб.: Энциклопедия. Большая Российская Энциклопедия, 1999.

9. Ронжин А. Ф., Точные формулы для вероятностных характеристик времени выхода накапливающихся сумм исходов бернуллиевской последовательности за прямолинейные границы и их применение к определению средней информационной скорости одного способа скрытой передачи информации. Обозрение прикладной и промышленной математики (2007) 14, №4, 593-597.

10. Оуэн Д. Б., Сборник статистических таблиц. ВЦ АН СССР, Москва, 1966.

11. Bahadur R. R., Some approximations to the binomial distribution. Ann. Math. Statist. (1960) 3, №1, 43-54.

12. Феллер В., Введение в теорию вероятностей и ее приложения. Мир, Москва, 1964.

13. Колчин В. Ф., Случайные отображения. Наука, Москва, 1984.

Статья поступила 21.01.2009. 